\title{
Absorption features of the zero frequency mode in an ultra-thin slab
}

\author{
Jose M. Llorens, ${ }^{\text {a) }}$ Jerónimo Buencuerpo, and Pablo Aitor Postigo \\ IMM-Instituto de Microelectrónica de Madrid (CNM-CSIC), Isaac Newton 8, PTM, E-28760 Tres Cantos, \\ Madrid, Spain
}

(Received 3 October 2014; accepted 28 November 2014; published online 9 December 2014)

\begin{abstract}
The optical absorption in a homogeneous and non-dispersive slab is governed by the well-known Fabry-Perot resonances. We have found that below the lowest order Fabry-Perot resonance, there is another absorption maximum due to the zero frequency mode whose peak frequency is given not by the real part of the complex resonance frequency, as it is the case for all other resonances, but by the imaginary part. This result is of interest, among other applications, for ultra thin solar cells, as tuning the zero frequency mode peak with the maximum of solar irradiance results in an increased efficiency. (C) 2014 AIP Publishing LLC. [http://dx.doi.org/10.1063/1.4904027]
\end{abstract}

The theoretical description of the optical properties of a homogeneous slab is a problem extendedly covered in optics and electromagnetics textbooks. Conceptually, it plays a central role in the design of solar cell devices, where the active region is constituted by a single homogeneous layer. ${ }^{1,2}$ Very recently, unexpected features on the absorption have been reported when a lossy layer is placed on top of a metallic substrate. ${ }^{3,4}$ In addition, inhomogeneous layers are frequently approximated by a homogeneous layer of effective material parameters through homogenization techniques. ${ }^{5}$ In the ultra-thin limit (small thickness to wavelength ratio), they accurately describe bidimensional materials, such as graphene. ${ }^{6}$ Aside of its potential application, it is an introductory problem to illustrate fundamental concepts such as field continuity conditions, interference, and phase delay. In the case of a slab comprising a non-dispersive material, the reflection and transmission spectra exhibit a series of maxima and minima which can be exactly described as an interference effect between the wave reflected or refracted by the two interfaces bounding the slab. These spectral features are the core of the Fabry-Perot (FP) interferometer and therefore are known as FP resonances. From the constructive interference condition, the maxima in the transmission spectrum follows a simple formula $\omega_{m}=c m \pi / n L$, being $c$ the light speed in vacuum, $n$ the material refractive index, $L$ the slab thickness, and the integer $m=1,2, \ldots$ the order of the FP resonance. If the material presents losses, these are described by a complex refractive index. The absorption spectra result in a series of peaks, where their maxima are coincident with those of the transmission spectra. However, the peak positions are not accurately predicted anymore by the FP formula, specially under strong losses. The illumination of a dielectric layer can be seen as a scattering problem, and hence the main features of the spectra can be attributed to excitation of resonances. System resonances can be found as the poles of the scattering matrix ${ }^{7}$ and, for the case of flat surfaces, as the poles of the reflection or transmission coefficient. ${ }^{8}$ Moreover, as noted by Xia et al. ${ }^{8}$ the pole condition for the reflection coefficient is equivalent to the guidance

\footnotetext{
${ }^{\text {a) }}$ Author to whom correspondence should be addressed. Electronic mail: jose.1lorens@csic.es
}

condition in the slab. A complete picture of the problem can be attained from an earlier work. Kliewer, Fuchs, and Pardee authored a thorough and detailed analysis of this problem in a series of papers. ${ }^{9-11}$ The authors introduce the concept of the light-line to distinguish between the radiative region (solution above the light-line in the dispersion diagram) and the non-radiative one (below the light-line). The latter are described by the normal modes emerging from Maxwell's equations while the former are treated as virtual modes, ${ }^{12}$ i.e., solutions for which the fields are non-zero away from the slab $(r \rightarrow \infty)$. Even though the study focusses on the particular physics of an ionic crystal slab (LiF), their main conclusions are valid to a dispersive slab. Indeed, the dispersion relations are the same as those found for a step-profile planar waveguide (Chapter 12, Ref. 13). Virtual modes are nowadays known as leaky modes in photonics ${ }^{14}$ and waveguide theory, ${ }^{13}$ but also as Mie resonances ${ }^{15}$ or morphologydependent resonances. ${ }^{16}$ A proper treatment has only been drawn in the theoretical framework of quasinormal modes (QNMs), see Ref. 17 for a review and references therein.

In this letter, we carefully study the absorption spectrum in a non-dispersive homogeneous slab. We show that at frequencies below that of the "fundamental" FP resonance [see Fig. 1(a)], the absorption does not go to zero in a trivial way. In contrast, a maximum can be clearly identified. Relying on the QNMs of a slab, ${ }^{18}$ it is possible to precisely get the position and damping of all the resonances. However, the correspondence with the absorption peaks can only be made for those with $\operatorname{Re}\left(\omega_{m}\right), m=1,2, \ldots$. The position of the maximum observed at smaller frequencies has no correspondence with the real part of any quasinormal mode frequency. More intriguing is the fact that the imaginary part of the zerofrequency mode, $\operatorname{Im}\left(\omega_{0}\right)$, predicts its position [see Fig. 1(c)]. In first place, we use approximative theories based on the QNM frequencies to reproduce the absorption spectrum of an homogeneous slab. ${ }^{19}$ Although a good description of the spectrum is obtained, they predict a trivial fade-out of the absorption in the low frequency region. Only by properly expanding the fields in the complete basis of the QNMs, ${ }^{20}$ the full spectrum can be reproduced. We will show later that the spectral tuning of this peak with the solar irradiance maximizes the ultimate efficiency of a very thin solar cell. 

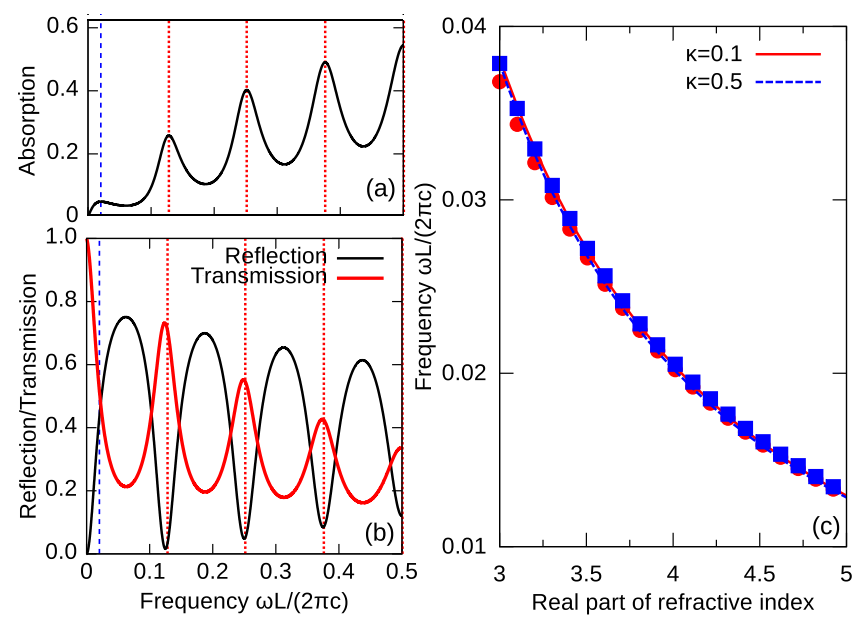

FIG. 1. Absortion (a) together with reflection and transmission (b) spectra for a layer of refractive index $N=4+i 0.1$. The vertical red dotted lines in (a) and (b) indicate the real part of the quasinormal mode frequencies, while the vertical blue dashed line indicates the imaginary part of the $m=0$ quasinormal mode frequency. Panel (c) shows the match between the imaginary part of the $m=0$ quasinormal mode frequency (solid symbols) with the maximum position derived by inspection of the exact spectrum (lines).

The absorption spectrum of a symmetric slab (same front and back refractive indices) is straightforward to calculate by invoking the principle of energy conservation and the Fresnel formulae. In Figure 1(a), we present such spectrum for a slab of refractive index $N=n+i \kappa=4+i 0.1$ in air. A series of symmetric peaks can be easily identified. Only the maximum close to zero frequency exhibits an asymmetric shape. To illustrate the correspondence with the transmission and reflection features, we show in panel (b) the complementary spectra. It is clear that at the frequencies of the symmetric absorption peaks, a maximum (minimum) in transmission (reflection) takes place. This is not the case for the asymmetric peak. A monotonous increase (decrease) of transmission (reflection) is found around the peak maximum. The frequencies of the QNMs can be derived from the dispersion relations of the guided modes. We will consider that the incoming light is in the $X Z$ plane, the slab is defined for $|x| \leq L / 2$, and the polarization is transversally electric. The dispersion relations read: $i k_{x 1} / k_{x 2}=\tan \left(k_{x 2} L / 2\right)$ for even modes and $-i k_{x 2} / k_{x 1}=\tan \left(k_{x 2} L / 2\right)$ for odd modes, where $k_{x j}=\sqrt{k_{j}^{2}-k_{z}^{2}}$ is the $x$ component of the wave vector in air (1) or slab (2). At normal incidence $\left(k_{z}=0\right)$ and for nondispersive material, it is easy to get the QNMs frequencies just by substituting $k_{x 2} / k_{x 1}=N$ in the dispersion relations. Even and odd solution can be combined into a single expression $^{21}$

$$
\tilde{\omega}_{m}=\frac{\omega_{m} L}{2 \pi c}=\frac{m}{2 N}-\frac{i}{2 \pi N} \ln \left(\frac{N+1}{N-1}\right)
$$

with $m=0, \pm 1, \pm 2, \ldots$ Even (odd) modes correspond to even (odd) $m$. Compact approximate expressions for the real and imaginary part of $\tilde{\omega}_{m}$ can be found in the limit $\kappa \ll n$ :

$$
\operatorname{Re}\left(\tilde{\omega}_{m}\right) \approx \frac{1}{2 n^{2}}\left\{m n-\frac{\kappa}{\pi}\left[\frac{2 n}{n^{2}-1}+\ln \left(\frac{n+1}{n-1}\right)\right]\right\},
$$

$$
\operatorname{Im}\left(\tilde{\omega}_{m}\right) \approx \frac{-1}{2 n^{2}}\left[m \kappa+\frac{n}{\pi} \ln \left(\frac{n+1}{n-1}\right)\right] .
$$

The red vertical lines in Figs. 1(a) and 1(b) denote $\operatorname{Re}\left(\tilde{\omega}_{m}\right)$ for $m \geq 0$. The position of the symmetric peaks is perfectly identified and corresponds to the well-known Fabry-Perot resonances. Only the asymmetric peak is left without QNM correspondence. However, $\operatorname{Im}\left(\tilde{\omega}_{0}\right)$ (indicated by a vertical dashed line) matches the spectral position of the maximum. This result can be generalized to other values of the refractive index as shown in Fig. 1(c). We have extracted the position of the maximum of the asymmetric peak by inspection of the absorption spectrum (lines) and superimposed to the value of $\operatorname{Im}\left(\tilde{\omega}_{0}\right)$ for two $\kappa$ values and as a function of $n$. The agreement between both results confirms that such prediction is not obtained by accident. In order to unravel the nature of this maximum and explain why a magnitude associated with the damping $[\operatorname{Im}(\omega)]$ predicts a peak position, we will proceed by exploring the contribution of each mode to the absorption.

Fuchs et al. provided a detailed analysis of the optical properties of a slab distinguishing between even and odd parity fields. ${ }^{11}$ The fact that the slab is illuminated only from one side breaks the mirror symmetry, and therefore, the incoming light can couple to even and odd QNMs. Indeed, the absorption can be decomposed into two non-interfering contributions: $A=\left(1-\left|P_{1}\right|^{2}\right) / 2+\left(1-\left|P_{2}\right|^{2}\right) / 2$, where $P_{1}$ and $P_{2}$ stand for an arbitrary complex amplitude of the even and odd fields, respectively. These amplitudes can be linearly expanded in terms of a complex frequency and the material damping $\kappa$. The absorption originated by the QNM $m$ results

$$
A_{m}=\frac{2 \Omega_{m}^{\prime \prime}\left(\omega_{m}^{\prime \prime}-\Omega_{m}^{\prime \prime}\right)}{\left(\omega-\omega_{m}^{\prime}\right)^{2}+\left(\omega_{m}^{\prime \prime}\right)^{2}}
$$

where $\omega_{m}=\omega_{m}^{\prime}+i \omega_{m}^{\prime \prime}$ and $\Omega_{m}=\Omega_{m}^{\prime}+i \Omega_{m}^{\prime \prime}$ are the QNM frequencies in presence $(\kappa \neq 0)$ and absence $(\kappa=0)$ of lossy media, respectively. The total absorption results of adding up the contribution of all QNMs. A comparison between the exact absorption spectrum and that obtained through Eq. (3) can be seen in Figure 2. The approximate solution reproduces quite accurately the FP resonances and goes to zero as $\omega \rightarrow 0$. The linear expansion is not capable to reproduce the low frequency peak. A better approach could be obtained by further expanding the fields to higher degree orders. However, as noted by Fuchs et al., ${ }^{11}$ increasing the complexity of the approximate model will eventually minder its illustrative potential, resulting in an effortless attempt.

Complementary, we have used the coupled mode theory (CMT), which is rooted in the coupling of modes. In general terms, the modes to be coupled can be either propagating or resonant. It results quite intuitive the description of how an incoming wave couples to the resonances of a cavity. ${ }^{22} \mathrm{CMT}$ has been employed in the description of a guide resonance in a photonic crystal slab, ${ }^{23}$ optical Fano resonances of nanostructures, ${ }^{24}$ and light absorption of low-dimensional semiconductor nanostructures. ${ }^{19}$ Following the derivation of $\mathrm{Yu}$ and $\mathrm{CaO},{ }^{19}$ the absorption due to the coupling with a QNM of a slab can be written as 

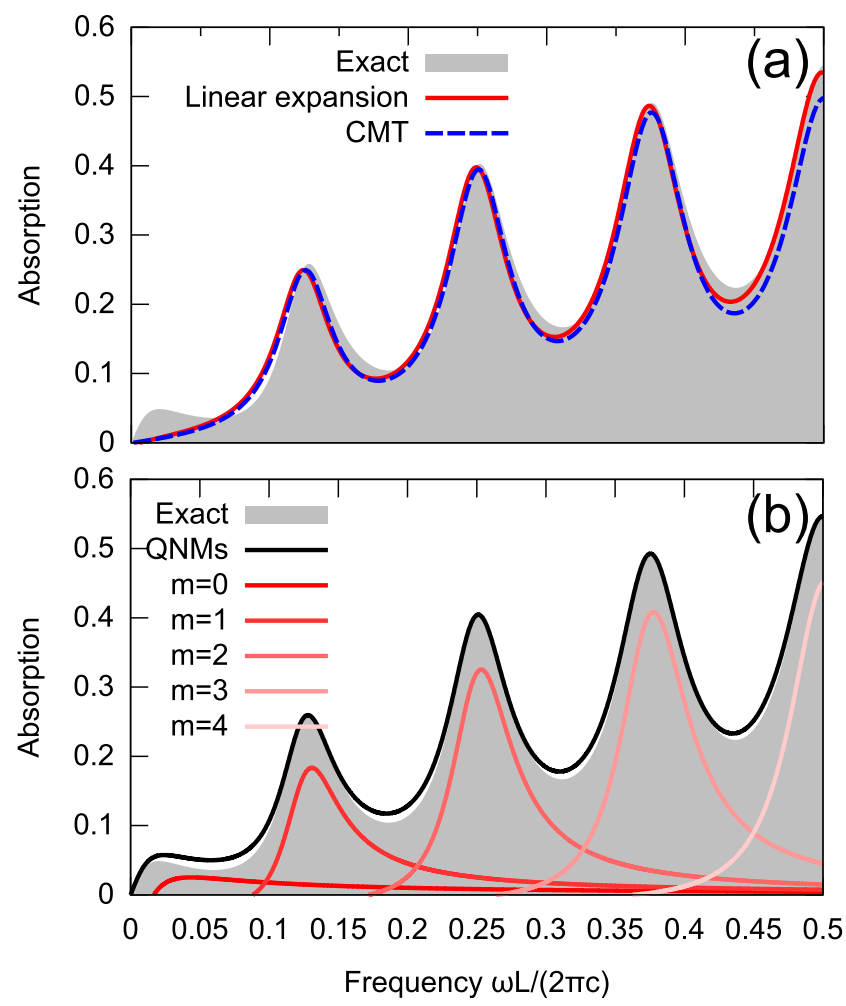

FIG. 2. (a) Comparison of the exact absorption spectrum to approximate solutions. Absorption from a linear expansion of the fields (red solid line) and coupled mode theory (blue dashed line). (b) Absorption spectrum obtained by computing the transmission with Eq. (5) and approximating the reflection with the value of $\tilde{E}(x, \omega)$ at $\mathrm{x} / \mathrm{L}=0.01$. The coloured lines represent the absorption considering only the QNM $m$. The shaded region is the exact solution.

$$
A_{m}=\frac{2 Q_{\mathrm{abs}}^{-1} Q_{\mathrm{rad}}^{-1}}{4\left(\omega / \omega_{m}-1\right)^{2}+\left(Q_{\mathrm{abs}}^{-1}+Q_{\mathrm{rad}}^{-1}\right)},
$$

where $Q_{\text {abs }}=n / 2 \kappa$ and $Q_{\text {rad }}=\omega_{m}^{\prime} / 2 \omega_{m}^{\prime \prime}$. Fig. 2(a) shows that the CMT also fails in reproducing the low frequency peak.

Finally, we introduce the QNM formalism with the aim of finding a complete basis of functions that allow for the expansion of the fields in an open cavity system. Completeness can be rigorously proved for a one dimensional cavity of refractive index $n(x)$ fulfilling (i) a discontinuity condition at the bounds of the cavity $[0, L]$ and (ii) the no tail condition, i.e., $n(x)=n_{0}$ for $x<0$ and $x>L .{ }^{20}$ Under this assumptions, the transmission of cavities such one dimensional photonic crystals has been studied. ${ }^{20,25}$ Extending the formalism to higher dimensions is rather involved. Some progresses have been made relying on numerical methods. ${ }^{26,27}$ The QNMs are defined by a set of eigenfrequencies [see Eq. (1)] and eigenfunctions $f_{m}(x)$. Considering the slab as a one dimensional open cavity illuminated from the left by an electric field $E_{\mathrm{p}}(x, t)$, the electric field in the cavity can be expanded in the basis of QNMs: $E(x, t)=\sum_{m} a_{m}(t) f_{m}(x)$. Applying the Fourier Transform, the field can be expressed in the frequency domain $\tilde{E}(x, \omega)=\sum_{m} \tilde{a}_{m}(\omega) f_{m}(x)$. Transmission can be exactly derived noting that the electric field at the right surface of the cavity is continuous with the transmitted field $\tilde{E}(L, \omega)=\tilde{E}_{\mathrm{T}}(L, \omega)$. Hence, $t(\omega)=\tilde{E}(L, \omega) / \tilde{E}_{\mathrm{p}}(0, \omega)$. The final expression results ${ }^{20}$

$$
t(\omega)=\frac{\sum_{m} \tilde{a}_{m}(\omega) f_{m}(L)}{\tilde{E}_{\mathrm{p}}(0, \omega)}=\zeta \sum_{m} \frac{(-1)^{m}}{\omega-\omega_{m}} .
$$

A similar procedure can be followed for the reflection coefficient

$$
r(\omega)=\frac{\tilde{E}(0, \omega)}{\tilde{E}_{\mathrm{p}}(0, \omega)}-1=\zeta \sum_{m} \frac{1}{\omega-\omega_{m}}-1 .
$$

The global factor is defined as $\zeta=2 i n_{0} c / \Delta n L$, where $\Delta n=n^{2}-n_{0}^{2}$. This expression fails in reproducing the exact reflection coefficient. Such disagreement results from the impossibility of the QNMs to cover the contribution of the incoming field. Note the different boundary conditions at the left and right surfaces of the cavity. This limitation remains unsolved although it has been reported by Ho et al. in Ref. 28. It is possible, however, to approximate the value of the field at $x=0$ by a value nearby within the cavity using the field expansion $\tilde{E}(x, \omega)$. The resulting absorption spectrum is shown in Fig. 2(b) together with the exact solution. In contrast with the spectra shown in Fig. 2(a), the peak appearing at low frequencies is accurately reproduced by the QNM theory. The main conclusion derived from this calculation is that the nature of such peak is well described by the set of QNMs. There is no need to explore for other type of Maxwell's solutions to describe its nature, like, for example, surface modes.

With the QNMs expansion, it is possible to get approximately the contribution of each mode to the absorption spectrum. In Fig. 2(b), we depict by colored lines the contribution of the $0 \leq m \leq 4$. The left-most peak gets naturally described by the $m=0$ order resonance. Although the coefficient of the $m=0$ mode appears as a Lorentzian-like function [see Eq. (5)] like any of the other modes $(m \neq 0)$, its shape in the absorption spectrum appears as an overdamped peak. This results from the fact that the limit of $L \rightarrow 0$ physically forces $R \rightarrow 0$ and $T \rightarrow 1$, hence the absorption must be zero. In addition, for $m=0$, it can be easily verified the overdamped condition from $\operatorname{Eq} .(2), \operatorname{Re}\left(\tilde{\omega}_{0}\right) / \operatorname{Im}\left(\tilde{\omega}_{0}\right)$ $\approx 2 \kappa / n \ll 1 .^{29}$ The position of the resonance is indeed given by $\operatorname{Re}\left(\tilde{\omega}_{0}\right) \approx 0$; however, it is of little interest given that the absorption maximum is notably displaced from this point. In Fig. 1(c), we show that the position of the absorption maximum is given by $\operatorname{Im}\left(\tilde{\omega}_{0}\right)$. Such finding results from the fact that the resonance is close to the $\omega \approx 0$. As the absorption must be zero there, the dominant contribution takes place at the right side of the peak. The peak width is given by $2 \operatorname{Im}\left(\tilde{\omega}_{0}\right)$. Hence, the maximum results to be located at $\operatorname{Re}\left(\tilde{\omega}_{0}\right)+\operatorname{Im}\left(\tilde{\omega}_{0}\right) \approx \operatorname{Im}\left(\tilde{\omega}_{0}\right)$. Thus, a spectral maximum position given by the imaginary part of the resonance frequency instead of its real part is a result certainly unexpected.

Hägglund et al. provide additional particularities of this absorption maximum by analysing the denominator of the transmission and reflection coefficients. ${ }^{5}$ They look for the value of the refractive index which maximizes the absorption value. Their analysis complements our findings; it is not only possible to determine the peak position but also the material properties which maximize the absorption. Moreover, 

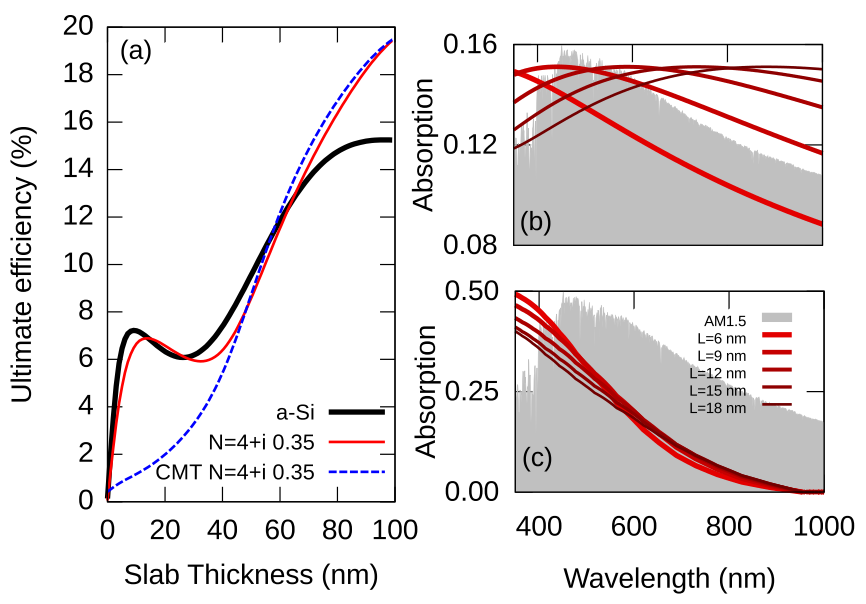

FIG. 3. (a) Ultimate efficiency of a bare slab for a non-dispersive material and for a-Si. Absorption spectra for different thicknesses of a non-dispersive (b) and a-Si (c) slab. The grey shaded area represents the AM1.5 solar spectrum.

Hägglund et al. show that the maximum is located at $n \approx \kappa$. In this limit, the permittivity is $\epsilon^{\prime}=n^{2}-\kappa^{2} \approx 0$, a regime characterized by a flat reflectivity and absorption. Only the zero-frequency mode survives for $L / \lambda \ll 1$.

Tayloring the position of the zero-frecuency peak is relevant for ultra-thin film solar cells, ${ }^{30,31}$ because the absorption limits the photocurrent and hence the efficiency. We have calculated the ultimate efficiency ${ }^{32}$ to illustrate the impact of the zero-frequency mode on a solar cell. We consider it as a single slab of a-Si. The results as a function of the slab thickness are shown in Fig. 3. The efficiency suffers a steep decrease as a result of the thinning of the slab. At very small thicknesses $(<20 \mathrm{~nm})$, a maximum appears. Such maximum can also be reproduced for the case of a nondispersive slab of refractive index $N=4+i 0.35$. It appears because of the spectral tuning of the zero-frequency peak maximum to the maximum of the solar irradiance AM 1.5. We also show in Fig. 3 the ultimate efficiency resulting from the computation of the absorption by the CMT. The contribution of such mode is certainly notable in this regime and must be accurately incorporated in any approximate solution employed for designing devices based on light absorption. In Fig. 3(b), we show the absorption spectra for thicknesses $L<20 \mathrm{~nm}$ on top of the AM 1.5 solar spectrum. As the thickness increases, the maximum redshifts. It results in a transition from a spectral match to a large detuning. For the actual a-Si material, such detuning is not obvious to identify [see Fig. 3(c)] as a result of the material dispersion. In particular, $\kappa$ in a-Si takes a high value at short wavelengths and diminishes monotonically towards longer wavelengths. It is, however, still possible to note the change in the slope of the absorption spectra as $L$ grows, which is related to the redshift of the peak maximum.

In summary, we have identified an absorption peak maximum which behaves differently from the well know FabryPerot resonances. It is not linked to a maximum in transmission and exhibits an asymmetric lineshape. We have presented three different methods to explore the absorption spectrum based on the structure of leaky modes or QNMs. Only the fully rigorous theory of QNMs was able to reproduce such maximum and explain its nature. Its position can be determined from the imaginary part of the zero-order QNMs frequency, which contrast with the common belief that only the real part contains information on peak positions. Finally, we have shown that for extremely thin slabs, such maximum is responsible of an increase in the ultimate efficiency making it roughly equal to that of a doubly thicker slab. When combined with other strategies for boosting the absorption in solar cells, e.g., light-trapping structures, it will contribute to increase the total efficiency of the device.

The authors acknowledge valuable discussions with Dr. Antonio García and Dr. Alessandro Settimi. In particular, the latter helped us in understanding the details of the QNMs expansion method. This work has been supported by Spanish MINECO (Grant Nos. ENE2012-37804-C02-02 and TEC2011-29120-C05-04).

${ }^{1}$ L. Yang, Y. Xuan, and J. Tan, Opt. Express 19, A1165 (2011).

${ }^{2}$ Z. Yu, A. Raman, and S. Fan, Proc. Natl. Acad. Sci. USA 107, 17491 (2010).

${ }^{3}$ M. A. Kats, R. Blanchard, P. Genevet, and F. Capasso, Nat. Mater. 12, 20 (2013).

${ }^{4}$ M. A. Kats and F. Capasso, Appl. Phys. Lett. 105, 131108 (2014).

${ }^{5}$ C. Hägglund, S. P. Apell, and B. Kasemo, Nano Lett. 10, 3135 (2010).

${ }^{6}$ J. R. Piper and S. Fan, ACS Photonics 1, 347 (2014).

${ }^{7}$ E. Popov, in Progress in Optics, edited by E. Wolf (Elsevier, 1993), Vol.

31, pp. 139-187.

${ }^{8}$ J. Xia, A. K. Jordan, and J. A. Kong, J. Opt. Soc. Am. A 9, 740 (1992).

${ }^{9}$ K. L. Kliewer and R. Fuchs, Phys. Rev. 144, 495 (1966).

${ }^{10}$ K. L. Kliewer and R. Fuchs, Phys. Rev. 150, 573 (1966).

${ }^{11}$ R. Fuchs, K. L. Kliewer, and W. J. Pardee, Phy. Rev. 150, 589 (1966).

${ }^{12}$ J. M. Blatt and V. F. Weisskopf, Theoretical Nuclear Physics (John Wiley \& Sons, Inc., 1952).

${ }^{13}$ A. W. Snyder and J. D. Love, Optical Waveguide Theory (Chapman \& Hall, 1991).

${ }^{14}$ K. Inoue and K. Ohtaka, Photonic crystals: Physics, Fabrication and Applications (Springer, 2004), Vol. 94.

${ }^{15}$ C. F. Bohren and D. R. Huffman, Absorption and Scattering of Light by Small Particles (John Wiley \& Sons, 2008).

${ }^{16}$ B. R. Johnson, J Opt. Soc. Am. A 10, 343 (1993).

${ }^{17}$ P. T. Kristensen and S. Hughes, ACS Photonics 1, 2 (2014).

${ }^{18}$ A. Settimi and S. Severini, J. Mod. Opt. 57, 1513 (2010).

${ }^{19}$ Y. Yu and L. Cao, Opt. Express 20, 13847 (2012).

${ }^{20}$ A. Settimi, S. Severini, and B. J. Hoenders, J Opt. Soc. Am. B 26, 876 (2009).

${ }^{21}$ The relation between tangent and natural $\log$ functions is: let $a=\tan z$, then $z=-i / 2 \ln [(i-a) /(i+a)]$.

${ }^{22}$ H. A. Haus, Waves and Fields in Optoelectronics (Prentice-Hall, Inc., Englewood Clifs, N.J., 1984).

${ }^{23}$ S. Fan, W. Suh, and J. D. Joannopoulos, J Opt. Soc. Am. A 20, 569 (2003).

${ }^{24}$ P. Fan, Z. Yu, S. Fan, and M. L. Brongersma, Nat. Mater. 13, 471 (2014).

${ }^{25}$ M. Maksimovic, M. Hammer, and E. van Groesen, Opt. Commun. 281, 1401 (2008)

${ }^{26}$ Q. Bai, M. Perrin, C. Sauvan, J.-P. Hugonin, and P. Lalanne, Opt. Express 21, 27371 (2013).

${ }^{27}$ C. Sauvan, J. P. Hugonin, I. S. Maksymov, and P. Lalanne, Phys. Rev. Lett. 110, 237401 (2013).

${ }^{28}$ K. C. Ho, P. T. Leung, A. Maassen van den Brink, and K. Young, Phys. Rev. E 58, 2965 (1998).

${ }^{29}$ The natural log must be approximated to its first order Taylor expansion: $\ln (x) \approx(x-1)$, valid only for $0<x<2$.

${ }^{30}$ R. Betancur, A. Martnez-Otero, X. Elias, P. Romero-Gmez, S. Colodrero, H. Miguez, and J. Martorell, Sol. Energy Mater. Sol. Cells 104, 87 (2012).

${ }^{31}$ Y. Park, E. Drouard, O. El Daif, X. Letartre, P. Viktorovitch, A. Fave, A. Kaminski, M. Lemiti, and C. Seassal, Opt. Express 17, 14312 (2009).

${ }^{32}$ J. Buencuerpo, L. E. Munioz-Camuniez, M. L. Dotor, and P. A. Postigo, Opt. Express 20, A452 (2012). 